\title{
Patientensicherheit gemeinsam fördern
}

\author{
Die Zusammenarbeit in und zwischen \\ den Krankenhäusern ist ausbaufähig
}

Wirtschaftliche Leistungsfähigkeit und hohe Versorgungsqualität und Patientensicherheit sind die Eckpunkte, die Europas Krankenhäuser vor große Herausforderungen stellen. Beim Kongress „Krankenhaus der Zukunft" diskutierten nationale und internationale Experten Ende Oktober in Wien mögliche Lösungen. Prozessmanagement im Krankenhaus der Zukunft und im Operationssaal von morgen bildeten die ganz konkreten Themenschwerpunkte und ein Joint Action Programm für Patientensicherheit und Versorgungsqualität für die europäische Zusammenarbeit wurde erstmals in Österreich präsentiert.

„Viele nosokomiale Infektionen, Medikationsfehler, Verwechslungen im OP oder Fehler bei Diagnose und Therapie wären vermeidbar, gebe es ausformulierte lokale, nationale und länderübergreifende Strategien zur Patientensicherheit“, stellte Dr. Brigitte Ettl, ärztliche Direktorin Krankenhaus Hietzing und Vorsitzende der Plattform Patientensicherheit fest." und Die Plattform Patientensicherheit fungierte gemeinsam mit der Initiative „Sicherheit im OP" und in Zusammenarbeit mit der Wirtschaftskammer Österreich-Initiative Plattform Gesundheitswirtschaft als Gastgeber der wissenschaftlichen Fachveranstaltung. Zwar gebe es, so Ettl, Bemühungen auf WHO-, EU- und nationaler Ebene, eine fehlende Gesamtstrategie habe allerdings zur Folge, dass die moderne Medizin stör- und fehleranfällig sei.

Der europäische Austausch ist daher wichtiger denn je. Das Joint Action Programm für Patientensicherheit und Versorgungsqualität (JA PASQ), das Jean Bacou, Koordinator des Europäischen Netzwerks für Patientensicherheit und Versorgungsqualität erstmals in Österreich vorstellte, soll künftig die Zusammenarbeit zwischen den 27 Europäischen Mitgliedstaaten, internationalen Organisationen und EU-Akteuren stärken.

Die Stärken und Fähigkeiten sowohl von öffentlichen als auch privaten Krankenhäusern besser zu nützen, forderte Mag. Julian Hadschieff, Obmann des Fachverbandes Gesundheitsbetriebe, CEO der PremiQaMed Unternehmensgruppe, sowie Mitinitiator der Plattform Gesundheitswirtschaft. Weniger Konkurrenz und mehr Ergänzung und gegenseitige Stärkung sollten ein konstruktives Miteinander ergeben.

\section{Effiziente Strukturen, zufriedene Mitarbeiter}

„Kostenersparnis und Effizienzgewinn dürfen nicht auf dem Rücken der Pflegekräfte erzielt werden", sagte Alexander Mainda, Bereichsleiter OP Dienste/OP Management an der Klinik Hirslanden

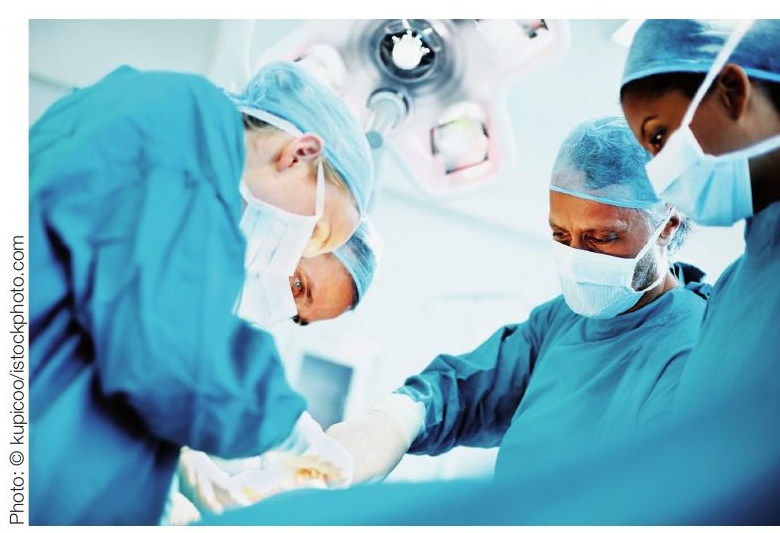

Wenn Arbeitsabläufe und Kommunikation im OP stimmen, steigt auch die Mitarbeiterzufriedenheit.

Zürich mit über 259 Betten und zwölf Operationssälen. Pro Jahr werden 12.000 Eingriffe durchgeführt. „Wenn die Arbeitsabläufe und die Kommunikation im OP stimmen, wenn das Umfeld ergonomisch und technisch auf dem neuesten Stand ist, steigt auch die Mitarbeiterzufriedenheit", berichtete Mainda aus der Praxis. „Kostendruck setzt oft Innovationen frei.“ Der Einsatz von OP-Trays habe maßgeblich zur Entspannung und Zeitersparnis in den Operationssälen der Schweizer Privatklinik beigetragen.

Kosten- und Zeiteinsparung bei höchster Sicherheit

Die Erhöhung der wirtschaftlichen Leistungsfähigkeit der Spitäler müsse mit der Steigerung der Sicherung der Versorgungsqualität einhergehen, forderte Stephan Schliack von der Initiative „Sicherheit im OP“. Kundenspezifische Einweg-OP-Sets könnten einen wichtigen Beitrag leisten, um diese Herausforderung zu meistern. Schliack: „Die kundenspezifischen Sets sparen Zeit und Kosten im OP sowie in den vorgelagerten Prozessen. $\mathrm{Zu}-$ dem fällt weniger Verpackungsmaterial an, da nahezu jede Einweg-Komponente unverpackt in ein Set integriert werden kann. Einweg-Sets sind außerdem sehr leicht und verfügen über ein geringes Volumen. Das erhöht die Lagerfähigkeit, spart Platz und reduziert das Transportvolumen."

Berechnungen der Fachhochschule Gelsenkirchen haben unter anderem ergeben, dass die Zeiteinsparungen in der Kommissionierung für den operativen Eingriff durch den Einsatz von OP-Trays zwischen 40 und 55 Prozent liegen.

Quelle: Presseaussendung wiko wirtschaftskommunikation 